\title{
REZENSIONEN
}

\section{Der Preis der deutschen Einheit}

Ritter, Gerhard A.: Der Preis der deutschen Einheit. Die deutsche Wiedervereinigung und die Krise des Sozialstaats, Beck-Verlag, München 2006, 540 Seiten, € 38,-.

Im Jahr 1994 stellte die Bundesregierung die ersten Überlegungen an, ein repräsentatives Forschungs- und Publikationsvorhaben zur Geschichte der Sozialpolitik im geteilten und wiedervereinigten Deutschland nach 1945 durchzuführen. Für die Herausgabe der auf 21 Bände ausgelegten Reihe, elf Bände zur Darstellung und zehn Dokumentenbände, zeichnen das Bundesministerium für Arbeit und Sozialordnung und das Bundesarchiv verantwortlich, die 2001 den ersten Band vorlegten. Für den letzten Doppelband 11 „Sozialpolitik im Zeichen der Vereinigung “ gewannen die Herausgeber den emeritierten Münchener Historiker Gerhard A. Ritter. Aus der Arbeit an dieser Reihe ist das hier anzuzeigende Buch hervorgegangen. Die genannten Herausgeber ermöglichten es Ritter, auf Akten und Dokumente zurückzugreifen, wie es in dieser Weise in der Regel erst nach Ablauf der Sperrfristen möglich ist. Davon profitiert das Buch in erheblicher Weise.

Mit Blick auf den Titel stehen zunächst zwei Fragen im Mittelpunkt der Untersuchung: Welchen Preis hatte die deutsche Einheit, und was hat das mit der Krise des Sozialstaats zu tun? Ritter macht bei der Antwort auf diese Fragen keine großen Umschweife. Die „schwere Krise“ des Sozialstaats in Deutschland „seit Anfang der 90er Jahre war ... wesentlich eine Folge der deutschen Vereinigung“" (S. 133). Die Ursachen hierfür sieht er in einer Fehleinschätzung der Schwierigkeiten seitens der politisch Verantwortlichen. Zu sehr war ihre Aufmerksamkeit durch die Außenpolitik absorbiert mit der Folge der finanziellen Überbeanspruchung des Systems. Im Überschwang der Ereignisse seien funktionierende Leistungssysteme wie das Gesundheitswesen der DDR voreilig durch ein System nach westdeutschem Vorbild ersetzt worden (S. 161 und S. 18 ff.). Insgesamt, so das Urteil Ritters, habe es aber keine andere realistische Option gegeben, die Vereinigung sozialstaatlich zu bewerkstelligen, als den westdeutschen Teilstaat nach 1989 institutionell und organisatorisch auf die fünf neuen Länder und Ostberlin auszudehnen. Die gewaltigen Probleme, die sich aus dem Zusammenbruch der politischen und sozialen Ordnung in der DDR ergaben, erforderten rasches Handeln. Es ist den politisch Verantwortlichen daher nicht vorzuwerfen, in der Hauptsache so wie skizziert verfahren zu sein.

Diese Phase der sich überschlagenden Ereignisse und der hastigen Weichenstellung erstreckte sich vom Fall der Mauer am 9. November 1989 bis zum Tag der Vereinigung am 3. Oktober 1990. In diese Zeit fällt die Wirtschafts-, Währungs- und Sozialunion, die für den bundesdeutschen Sozialstaat insgesamt von mindestens so großer Tragweite war wie die staatliche Wiedervereinigung.

Der Weichenstellung der ersten Phase folgte in der zweiten Phase, die sich laut Ritters überzeugender Periodisierung vom 3. Oktober 1990 bis zum März 1992 erstreckte, die 
Überleitung des westdeutschen Sozialsystems und -rechts einschließlich der Arbeits-, Verwaltungs- und Sozialgerichtsbarkeit. Angesichts der Größe und Bedeutung der Aufgabe gesteht Ritter den Akteuren zu, in der mittleren Frist im Großen und Ganzen erfolgreich agiert zu haben. Besonders hebt er hervor, dass der Vereinigungsprozess ohne größere politische und soziale Verwerfungen vonstatten ging. Die weitgehend pünktliche und zutreffende Auszahlung der Renten nach dem Rentenüberleitungsgesetz stellt eine der beeindruckendsten Leistungen der Sozialunion zwischen Ost und West dar.

Ungeachtet dessen müsse aber der Vorwurf erhoben werden, dass die Vereinigung besonders auf dem Gebiet des Sozialen unsolide finanziert worden ist. Die strikte Weigerung des Bundesfinanzministers Theo Waigel, die Haushaltsrisiken der Vereinigung dem Bundeshaushalt aufzubürden, führte dazu, dass der Finanzausgleich Richtung Osten über das Sozialsystem abgewickelt wurde (S. 253 ff.). Durch diesen Befund kann Ritter begründen, worin der „Preis der deutschen Einheit“ bestanden hat. Die unsolide Finanzierung der deutschen Einheit über die Sozialversicherungssysteme habe zur Folge gehabt, dass die in den achtziger Jahren ohnehin zögerliche Reformpolitik für einige Jahre unterbrochen wurde, wenn nicht sogar Rückschritte zu verzeichnen waren. Ursächlich hierfür sei die Entscheidung gewesen, die Vereinigung über Schulden und steigende Sozialversicherungsbeiträge zu finanzieren, was die im globalen und im europäischen Wettbewerb stehende deutsche Wirtschaft erheblich belastet habe. Dafür verantwortlich zu machen seien Bundeskanzler Helmut Kohl, der das Versprechen abgegeben hatte, wegen der Wiedervereinigung müssten die Steuern nicht erhöht werden, und Finanzminister Theo Waigel von der CSU, der dem Bundeskanzler bei dieser Politik treu zur Seite stand. Und im Einklang damit schloss Bundessozialminister Norbert Blüm Leistungskürzungen aufgrund der Vereinigung schon im Herbst 1990 kategorisch aus (S. 266).

Bereits im Frühjahr 1992 wurde jedoch offenkundig, dass der eingeschlagene Weg in die Sackgasse führte. Die tiefe Rezession der Jahre 1993/94 war nicht zuletzt Folge der vorausgegangenen Fehlentscheidung zur Finanzierung der deutschen Einheit. Die im föderalen Konsolidierungsprogramm und in den Haushaltssicherungsgesetzen 1993 und 1994 getroffenen Maßnahmen bedeuteten tiefe Einschnitte in die staatlichen und sozialen Leistungen, die die Regierung Kohl / Kinkel 1994 beinahe die Regierungsgewalt gekostet hätten.

Dazu passt allerdings gar nicht die Einführung der Pflegeversicherung, die Sozialminister Blüm 1994 mit dem Gewicht seiner Person und seines Amtes gegen vielfältige Widerstände durchsetzte. Dieser Erfolg verweist auf ein anderes Phänomen dieser Jahre der Wiedervereinigung. In dieser Zeit war der Einfluss der Verbände und der Länder auf die Politik vergleichsweise gering (S. 281 - 292). Wie in der Außen- und Deutschlandpolitik waren die Jahre 1989 bis 1994 auch in der Sozialpolitik Jahre der Exekutive. So ergibt sich das Bild eines handlungs- und durchsetzungsfähigen Staates, das sich nicht in die modernen Abgesänge auf den Staat einfügen lässt.

Ritter kommt zu dem Ergebnis, dass die Vereinigungspolitik auf dem Feld des Sozialen, nicht zuletzt mangels Alternativen, mit kleineren Einschränkungen erfolgreich war und eine große Leistung darstellt, soweit es die institutionelle, organisatorische und rechtliche Seite angeht. Im Hinblick auf die Finanzierung fällt sein Urteil allerdings eindeutig und hart aus: mangelhaft.

Über diese die großen Linien der Politik betreffende Darstellung weist Ritter noch auf eine Reihe Details hin, die für die Sozialpolitik im Vereinigungsprozess von Interesse sind und Hinweise darauf geben, dass sich in der mittleren und langen Frist auf Grund von Ent- 
täuschungen Verdruss über den Sozialstaat einstellen könnte. Ein Beispiel ist die Überleitung des Arbeitsrechts, die gleich mehrfach Probleme verursachte. Das deutsche Arbeitsrecht ist in der Hauptsache Richterrecht und demzufolge vergleichsweise zersplittert. Daraus resultierte Rechtsunklarheit auf Seiten der Arbeitgeber wie Arbeitnehmer, was in einer Bevölkerung, die Arbeitslosigkeit nicht kannte, zu erheblicher Verunsicherung führte. Vielfach entstand der Eindruck, der Staat überlasse den Einzelnen ungeschützt seinem Schicksal. Hinzu kam, dass der Aufbau der Arbeitsgerichtsbarkeit recht langsam vorankam. In der zweiten Instanz überstieg erst 1994 die Zahl der Erledigungen die der neu eingegangenen Klagen.

Weitere Beispiele ließen sich anführen. Sie weisen darauf hin, dass der Aufbau des Sozialstaats in Ostdeutschland aus der Vogelperspektive unbestritten eine beachtliche politische Leistung darstellt, aus der Froschperspektive vieler Betroffener jedoch Demütigung und Scheitern bereithielt. Die Enttäuschung lässt sich an der hohen Zahl der Nichtwähler beziehungsweise an den Wahlergebnissen für NPD, DVP oder PDS (jetzt Linkspartei) ablesen. Welcher Sprengsatz hier schlummert, ist nicht abzusehen. Wer etwas über einige der Gründe erfahren möchte, woher die Enttäuschungen rühren, wird bei Ritter hinlänglich fündig. Es ist folglich eine Studie anzuzeigen, die nicht nur eine fundierte, quellengesättigte und im Urteil sowohl abgewogene als auch sichere historische Darstellung enthält, sondern zur Beurteilung der Gegenwart wichtige Aufschlüsse liefert. „Der Ritter“ dürfte auf Jahre hinaus das Standardwerk zur Sozialpolitik der Wiedervereinigung sein.

Stefan Schieren

\section{Das rot-grüne Projekt}

Egle, Christoph und Reimut Zohlnhöfer (Hrsg.): Ende des rot-grünen Projektes. Eine Bilanz der Regierung Schröder 2002-2005, VS Verlag für Sozialwissenschaften, Wiesbaden 2007, 540 Seiten, $€ 34,90$.

Anfang und Ende der rot-grünen Bundesregierung haben sich mit spektakulären Bildern eingeprägt. Den Wahlsieg 1998 feierte das Führungstrio Gerhard Schröder, Joschka Fischer und Oskar Lafontaine mit seinen Anhängern in ausgelassener Stimmung. Der Machtwechsel wurde inszeniert als Generationswechsel, mit dem ein neuer Politikstil in der „Berliner Republik“ Einzug halten sollte. 2005 endete der Wahlabend mit dem ebenso denk- wie fragwürdigen Auftritt des noch amtierenden Kanzlers Schröder in der Berliner „Elefantenrunde“. Doch was bleibt an politischer Substanz nach zwei Legislaturperioden Rot-Grün?

Christoph Egle und Reimut Zohlnhöfer ziehen in dem von ihnen herausgegebenen Band eine kritische Bilanz der Jahre 2002 bis 2005. Das über 500 Seiten starke, mit zahlreichen Tabellen und Grafiken angereicherte Buch schließt an die 2003 publizierte Zwischenbilanz zur ersten Amtszeit Schröders an. ${ }^{1}$ Diese hatte sich vor allem auf die Frage konzentriert, in-

1 Christoph Egle / Tobias Ostheim / Reimut Zohlnhöfer (Hrsg.), Das rot-grüne Projekt. Eine Bilanz der Regierung Schröder 1998-2002, Wiesbaden 2003. Vgl. dazu auch die Rezension von Everhard Holtmann, Sachkundige (Zwischen-)Bilanz der rot-grünen Bundesregierung, in: ZParl, 35. Jg. (2004), H. 2, S. 363 f. 